\title{
Prenatal diagnosis in factor XIII-A deficiency
}

\author{
Caroline J Killick, Carol J Barton, Shazia Aslam, Graham Standen
}

\begin{abstract}
Congenital factor XIII deficiency is a severe bleeding disorder that is inherited as an autosomal recessive trait. The condition is commonly due to absence of the factor XIII-A subunit protein in the plasma. The case of a baby is reported who showed typical clinical features of factor XIII-A deficiency, including recurrent bleeding from the umbilical stump and a life threatening haemorrhage after circumcision. Family studies were performed and molecular analysis, using a Short Tandem Repeat (STR) marker closely linked to the A subunit gene, allowed antenatal exclusion diagnosis to be undertaken in a subsequent pregnancy. The case highlights the importance of seeking a family history of bleeding disorders before surgery in the neonatal period, particularly if the parents are consanguineous.

(Arch Dis Child Fetal Neonatal Ed 1999;80:F238-F239)
\end{abstract}

Keywords: factor XIII-A deficiency; prenatal diagnosis; autosomal recessive trait

Congenital factor XIII deficiency is a rare inherited bleeding disorder that commonly occurs in neonates. ${ }^{1}$ We report a case of a baby with this disorder who developed a large sub-aponeurotic haemorrhage when 2 days old, with recurrent bleeding from the umbilical stump and a life-threatening haemorrhage after a circumcision on day 28. After the coagulation defect had been diagnosed, genetic studies were undertaken which ensured that a successful prenatal diagnosis was performed in a subsequent pregnancy.

\section{Case history}

A baby was born at 39 weeks of gestation to a 21 year old primagravid woman of Pakistani origin. The parents were second cousins. One of the mother's siblings had died in infancy as a result of superficial haemorrhaging from the penis following circumcision. Two further cousins were receiving treatment for factor XIII deficiency. There was also a family history of congenital adrenal hyperplasia.

The infant was delivered by spontaneous vaginal delivery and had received $500 \mathrm{mcg}$ of intramuscular vitamin $\mathrm{K}$ immediately after birth. At 24 hours of age he had a large sub-aponeurotic haematoma which had extended by day 3 to involve much of the left side of the face. The blood picture showed that haemoglobin was $95 \mathrm{~g} / \mathrm{l}$, with a platelet count of $170 \times 10^{9} / 1$; International Normalised Ratio (INR) 1.0, APTR 1.04. An $x$-ray of the skull showed no evidence of fracture and a cranial ultrasound scan revealed no intracranial haem- orrhage. The infant received a blood transfusion and a second dose of vitamin $\mathrm{K} 500 \mathrm{mcg}$. $\mathrm{He}$ was discharged home at 6 days of age.

At 18 days of age, he was referred with umbilical bleeding, thought to be due to an umbilical granuloma. His clotting studies were again normal. At 28 days a circumcision was performed by a General Practitioner. Over the next 48 hours the wound intermittently haemorrhaged, the clot separating each time the dressings were changed. On admission at 30 days old, physical examination revealed a pale, well grown infant with a tachycardia of 160 beats per minute. No other abnormalities were detected. Investigations showed that his haemoglobin was $40 \mathrm{~g} / 1$, INR 1.18 , and APTR 1.33. Clotting assays revealed a factor VIII value of $2130 \mathrm{U} / 1$ and a factor IX concentration of $390 \mathrm{U} / 1$ (both normal for age). Initial testing using the $1 \%$ monochloroacetic acid test for clot stability suggested factor XIII deficiency. A factor XIII-A activity of $4.5 \%$ was later confirmed by chromogenic assay (normal neonatal range: $50-60 \%$ ). The activity detected was probably due to transfusion in the neonatal period. The mother's factor XIII-A value was $66 \%$ and the father's $69 \%$ (normal range in adults: $70-140 \%$ ). The infant's cranial ultrasound appearances remained normal. He received a further blood transfusion and monthly prophylactic treatment with factor XIII concentrate. This has successfully prevented further haemorrhaging and his development to date is normal.

Molecular genetic studies were performed in this family to allow first trimester antenatal exclusion diagnosis to be undertaken in a subsequent pregnancy. A highly polymorphic Short Tandem Repeat (STR) sequence, HUMF13A01 (AAAG)n, in the immediate 5' untranslated region of the A subunit gene, was detected using polymerase chain reaction (PCR). ${ }^{2}$

Leucocyte DNA was isolated from the parents and the proband; a chorionic villus sample taken at 11 weeks was used as a source of fetal DNA. The oligonucleotide primers described by Polymeropoulos and coworkers ${ }^{3}$ were used: sense strand 5' GAGGT TGCACTCCAGCCTTT 3' and antisense strand 5'ATGCCATGCAGATTAGAAA 3'. The PCR conditions used were denaturation $94^{\circ} \mathrm{C}$ for 1 minute, annealing at $50^{\circ} \mathrm{C}$ for 1 minute, and primer extension at $73^{\circ} \mathrm{C}$ for 1 minute. After 30 cycles of amplification and a final extension step of $73^{\circ} \mathrm{C}$ for 10 minutes, the products were run on a $6 \%$ polyacrylamide gel $(1 \times \mathrm{TBE}$ buffer $)$ and visualised under ultraviolet light after staining with ethidium bromide.

The results of the analysis are shown in fig 1. The father $\left(\mathrm{I}_{2}\right)$ is homozygous $\mathrm{L} 1 / \mathrm{L} 1$ for the 


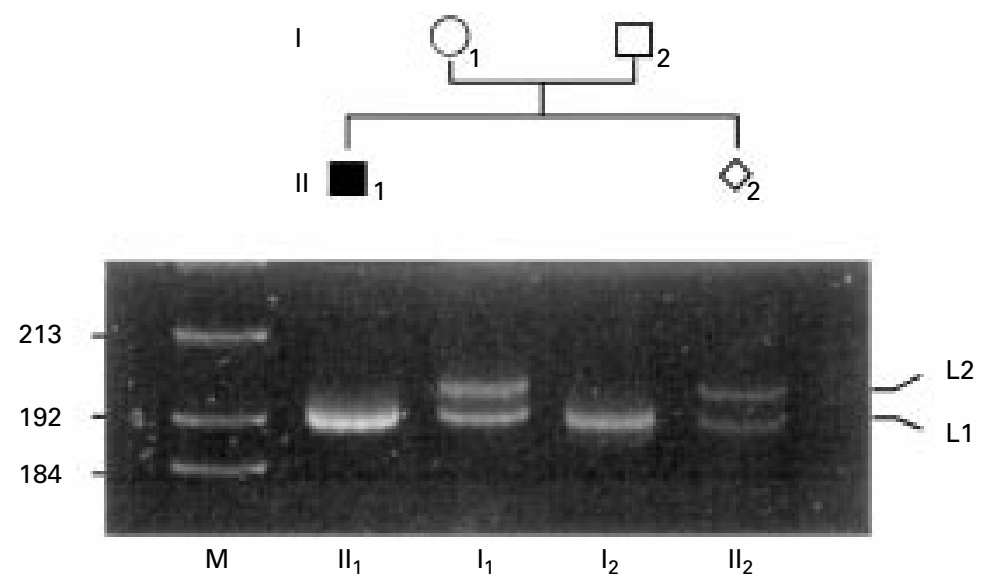

Figure 1 Antenatal exclusion diagnosis of factor XIII-A deficiency using the STR sequence HUMF13A01. M: molecular size markers (base pair) pBR322 digested with HaeIII. most cases the mutation is family specific. ${ }^{7}$ Missense mutations are most frequently detected with most amino acid substitutions located in the highly conserved catalytic core domain of the A subunit protein. ${ }^{8}$ Other defects, including microdeletions, frameshift, and nonsense mutations, are randomly distributed through the genomic sequence. ${ }^{9} 10$ If the location of the specific mutation is unknown, we have shown that the closely linked polymorphic marker HUMF13A01 can provide a valuable tool for gene tracking and accurate prenatal diagnosis. The size of this (AAAG)n tetra nucleotide repeat sequence can be rapidly and simply determined using PCR amplification. Previous studies have shown that the heterozygosity rate is around $75 \%$ in several ethnic groups and because it is located in the immediate 5 ' region of the gene, the risk of meiotic recombination is negligible. Kangsadalampai et $a l^{11}$ have recently studied the segregation of the STR alleles in seven families with A subunit deficiency and showed that, in all cases, the inheritance of the mutated allele could be unequivocally linked to the STR marker.

This case highlights the importance of seeking a medical and family history of bleeding disorder in neonates before surgery. Haemophilia $\mathrm{A}$ and $\mathrm{B}$ are the commonest inherited coagulation disorders which may present with a severe haemorrhagic tendency in early life. However, rare autosomal recessive conditions including factor XIII deficiency, type III von Willebrand disease, and congenital afibrinoginaemia may also be encountered, particularly in the offspring of consanguineous parents.

We are grateful to Dr PLF Giangrande of the Oxford Haemophilia Centre for his advice and assistance in the management of this case. The CVS was kindly performed by $\mathrm{Mr}$ Linda Tyfield, Southmead Hospital, Bristol, for assistance with fetal DNA extraction.

1 Board PG, Losowsky MS, Miloszewski KJA. Factor XIII: inherited and acquired deficiency. Blood Rev 1993;7:22942 .

Puers C, Holly A, Hammond C, et al. Allelic ladder characterisation of the short tandem repeat polymorphism in the 5' flanking region to the human coagulation factor XIII A subunit gene. Genomics 1994;23:260-4

3 Polymeropoulos MH, Rath DS, Xiao H, Merril CR. Tetranucleotide repeat polymorphism at the human coagulation factor XIII A subunit gene (F13A1). Nucleic Acids Res 1991;19:4306.

4 Chung SI, Lewis MS, Folk JE. Relationships of the catalytic properties of human plasma and platelet transglutaminase (activated blood coagulation factor XIII) to their subunit structures. F Biol Chem 1974;249:940-50.

5 Hashiguchi T, Saito M, Morishita E, et al. Two genetic defects in a patient with complete deficiency of the $b$ subunit for a pationt with complete deficiency of the

6 I sinose A, Davie EW. Characterisation of the subunit of human factor XIII (plasma transglutaminase), a blood coagulation factor. Proc Natl Acad Sci USA 1988;85:5829-33.

7 Anwar R, Stewart AD, Miloszewski KJA, et al. Molecular basis of inherited factor XIII deficiency: identification of multiple mutations provides insights into protein function. Br F Haematol 1995;91:728-35.

8 Aslam S, Yee VC, Narayanan S, et al. Structural analysis of a missense mutation (Val414Phe) in the catalytic core domain of the factor XIIIA subunit. $\mathrm{Br} \mathcal{F}$ Haematol 1997;98:346-52.

9 Aslam S, Bowen DJ, Mandalaki T, et al. Factor XIIIA subunit deficiency due to a homozygous 13-base pair deletion in exon 3 of the A subunit gene. Am 7 Hematol 1996;53:77-80

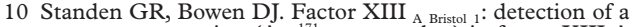
nonsense mutation ( $\mathrm{Arg}^{171} \rightarrow$ stop codon) in factor XIII A subunit deficiency. Br f Haematol 1993;85:769-72.

11 Kangsadalampai S, Coggan M, Caglayan SH, et al. Application of HUMF13A01 (AAAG)n STR polymorphism to the genetic diagnosis of coagulation factor XIII deficiency. Thromb Haemostas 1996;76:879-82. 